\title{
Research Article Symmetry Feature and Construction for the 3-Band Tight Framelets with Prescribed Properties
}

\author{
Jianjun Sun, ${ }^{1}$ Bin Huang, ${ }^{2}$ Xiaodong Chen, ${ }^{2}$ and Lihong Cui ${ }^{2}$ \\ ${ }^{1}$ College of Chemical Engineering, Beijing University of Chemical Technology, Beijing 100029, China \\ ${ }^{2}$ School of Science, Beijing University of Chemical Technology, Beijing 100029, China \\ Correspondence should be addressed to Lihong Cui, lhongcui@163.com
}

Received 5 July 2012; Accepted 29 August 2012

Academic Editor: Jingxin Zhang

Copyright (C) 2012 Jianjun Sun et al. This is an open access article distributed under the Creative Commons Attribution License, which permits unrestricted use, distribution, and reproduction in any medium, provided the original work is properly cited.

A construction approach for the 3-band tight wavelet frames by factorization of paraunitary matrix is developed. Several necessary constraints on the filter lengths and symmetric features of wavelet frames are investigated starting at the constructed paraunitary matrix. The matrix is a symmetric extension of the polyphase matrix corresponding to 3-band tight wavelet frames. Further, the parameterizations of 3-band tight wavelet frames with $3 N+1$ filter lengths are established. Examples of framelets with symmetry/antisymmetry and Sobolev exponent are computed by appropriately choosing the parameters in the scheme.

\section{Introduction}

In the theory and applications of wavelets and wavelet frames, certain properties are always desirable. It is well known that symmetry and high vanishing moments are very important features of all wavelets. Symmetry, which is also called linear phase in the language of engineering, is claimed to improve the rate-distortion performance in image compression $[1,2]$. On the one hand, parameterizations of FIR systems are of fundamental importance to the design of filters with the desired properties [1,3]. On the other hand, the advantages of MRA-based tight wavelet frames and their promising features in applications have attracted a great deal of interest and effort in recent years to extensively study them (e.g., [4-15]). The main tools for construction and characterization of wavelet frames are the unitary extension principle (UEP) [16] and its versions generalized such as OEP and MEP [17]. They give sufficient conditions for constructing MRA-based tight and dual wavelet frames. Many authors have worked on the design of wavelet frames with good properties. Most deals with 2-band wavelet frames systems, and a few authors have studied $M$-band framelets [8]. $M(\geq 2)$-band wavelets have advantages over dyadic wavelets in some aspects. For example, 
it enables a finer frequency partitioning and can provide a more compact representation of signals [18-21].

This paper deals with the construction of 3-band tight wavelet frames filters with prescribed properties using factorization and parameterizations of the paraunitary matrices. The parameter space describing 3-band wavelets is much richer than that in 2-band case; thus, it has greater freedom and flexibility. Concretely, with the describing of unitary extension principle (UEP) in the polyphase representation, we firstly construct a paraunitary matrix based on the polyphase matrix corresponding to compactly supported wavelet frames with the least number generators. Further, we establish necessary constraints on the filter lengths and symmetric features of wavelet frames. Then, we investigate the parameterizations of 3band tight wavelet frames with $3 N+1$ filter lengths. Finally, examples of 3-band wavelet frames with symmetry/antisymmetry and good smoothness are given by applying the proposed scheme.

Throughout this paper, let $N$ and $Z$ denote the sets of all natural numbers and integers, respectively. Let $A^{*}, A^{T}$, and $\operatorname{Tr}(A)$ denote conjugate transpose, transpose and the trace of $A$, respectively. Let $A=\left[a_{i j}\right]_{m \times n}, 1 \leq i \leq m ; 1 \leq j \leq n$ denote the $m \times n$ matrix with elements $a_{i j}$, for convenience, we omit the subscript $m \times n$ when $m=n$. Let $I_{n}$ denotes the $n \times n$ identity matrix and $J_{n}$ denote the $n \times n$ exchange matrix with ones on the antidiagonal. For $s \geq 0$, we use $W^{s}(R)$ denote the Sobolev space consisting of all functions $f$ with $\left(1+|\omega|^{2}\right)^{s / 2} \widehat{f}(\omega) \in L^{2}(R)$. In this paper, we only consider compactly supported wavelet frame and causal sequence with real finite impulse response (FIR), such sequences can be identified with Laurent polynomial defined by $h(z)=\sum_{k=0}^{K} h_{k} z^{-k}$, where 0 and $K$ are the smallest and largest indices that $h_{k}$ is nonzero, respectively. Assumpsit: $h(\omega)=h(z)$ and $z=e^{i \omega}$. We use leng $(h)=K+1$ to denote the filter length of $h$.

\section{UEP of Tight Framelets in Terms of Polyphase Representation}

In this section, we introduce some notation and state needed results that will be used later in this paper. Let refinable functions $\psi^{0}$ with dilation factor 3 generate a multiresolution analysis (MRA) $\left\{V_{j}\right\}_{j \in Z}$ of $L^{2}(R)$ and $\Psi=\left\{\psi^{1}, \ldots, \psi^{r}\right\} \subset V_{1}$. Then for real-value sequence $h_{\ell, k}, \psi^{\ell}$, $\ell=0,1,2, \ldots, r$ satisfy

$$
\psi^{\ell}(x)=3 \sum_{k \in Z} h_{\ell, k} \psi^{0}(3 x-k)
$$

or equivalently

$$
\widehat{\psi}^{\ell}(3 \omega)=h_{\ell, k}(\omega) \widehat{\psi}^{0}(\omega),
$$

where $h_{\ell}(\omega)=\sum_{k \in Z} h_{\ell, k} e^{-i k \omega}$ or rewritten $h_{\ell}(z)=\sum_{k \in Z} h_{\ell, k} z^{-k}, z=e^{-i \omega} . h_{0}$ is called a refinement mask or the low filter, and $h_{\ell}, \ell=1,2, \ldots, r$ are called wavelet masks or the high pass filters of the system, respectively. For notational convenience, refinement mask together with wavelet masks $\left\{h_{0}, h_{1}, \ldots, h_{r}\right\}$ is also called combined MRA masks in [7]. 
For given $\Psi=\left\{\psi^{1}, \ldots, \psi^{r}\right\} \subset L^{2}(R)$, define the wavelet system as $X(\Psi)=\left\{\psi_{j, k^{\prime}}^{\ell} \ell=\right.$ $1, \ldots, r ; j, k \in Z\}$, where $\psi_{j, k}^{\ell}=3^{j / 2} \psi^{\ell}\left(3^{j} x-k\right), j, k \in Z$.

Definition 2.1. The system $X(\Psi)$ is called an MRA-based 3-band tight wavelet frames of $L^{2}(R)$ if

(1) $\Psi \subset V_{1}$,

(2) $X(\Psi)$ is tight wavelet frames, that is,

$$
\|f\|_{L^{2}(R)}^{2}=\sum_{\ell=0}^{r} \sum_{j \in Z} \sum_{k \in Z}\left|\left\langle f, \varphi_{j, k}^{\ell}\right\rangle\right|
$$

holds for all $f \in L^{2}(R)$. Furthermore, elements $\psi_{j, k^{\prime}}^{\ell} \ell=1, \ldots, r$ are said to be framelets.

If we introduce the following two block matrices: modulation matrix

$$
M(\omega)=\left[h_{\ell}\left(\omega+\frac{2 k \pi}{3}\right)\right]_{0 \leq \ell \leq r, 0 \leq k \leq 2},
$$

and polyphase matrix

$$
P(z)=\left[p_{\ell, n}(z)\right]_{0 \leq \ell \leq r, 0 \leq n \leq 2^{\prime}}
$$

where

$$
p_{\ell, n}(z)=\frac{1}{\sqrt{3}} \sum_{k \in Z} h_{\ell, 3 k+n} z^{-k}, \quad \ell=0, \ldots, r, n=0,1,2, z=e^{i \omega}
$$

then we describe the UEP for 3-band tight wavelet frames in terms of modulation matrix and polyphase matrix as follows.

Lemma 2.2. Let $\psi^{0} \in L^{2}(R)$ be a compactly supported refinable function generated by finitely supported refinement mask $h_{0}$ with $\widehat{\psi}^{0}(0)=1$. Then for combined MRA masks $\left\{h_{0}, h_{1}, \ldots, h_{r}\right\}$, the system $X(\Psi)$, where $\Psi=\left\{\psi^{1}, \ldots, \psi^{r}\right\}$ defined by (2.1) or (2.2) forms an MRA-based 3-band tight wavelet frame of $L^{2}(R)$ provided one of the following hold for $\omega \in[-\pi, \pi]$.

(a) Modulation matrix $M(\omega)$ is paraunitary, that is $M^{*}(\omega) M(\omega)=I_{3}$.

(b) Polyphase matrix $P(z)$ is paraunitary, that is $P^{*}(z) P(z)=I_{3}$.

Symmetry and high vanishing moments are very important features of all wavelets.

Definition 2.3. A casual FIR filter $q(z)=\sum_{k=0}^{K} q_{k} z^{-k}$ is called symmetric/antisymmetric, if $z^{-K} q\left(z^{-1}\right)=t q(z), t= \pm 1$, where $K / 2$ is called the center of $q(z)$. 
Lemma 2.4. Suppose that the mask $h_{\ell}(z)=\sum_{k=0}^{K_{\ell}} h_{\ell, k} z^{-k}$ is symmetric/antisymmetric, then $\psi^{\ell}$ defined by (2.1) is symmetric/antisymmetric about $K_{\ell} / 2$, respectively, that is, $\psi^{\ell}\left(K_{\ell}-x\right)=t \psi^{\ell}(x)$, where $\ell=0,1,2, \ldots, r$.

The vanishing moments of wavelets is related to the order of sum rules.

Definition 2.5. The refinement mask $h_{0}(\omega)$ has the sum rules of order $p$, if

$$
\left.h_{0}(\omega)\right|_{\omega=0}=1,\left.\quad h_{0}^{(m)}(\omega)\right|_{\omega=2 l \pi / 3}=0, \quad l=1,2, \quad m=0,1, \ldots, p-1,
$$

or equivalently

$$
\sum_{k} \xi^{k} k^{m} h_{0, k}=0, \quad m=0,1, \ldots, p-1, \xi=e^{2 \pi i / 3}
$$

Lemma 2.6. Suppose that the wavelet mask $h_{\ell}(\omega)$ satisfies $\left.h_{\ell}^{(m)}(\omega)\right|_{\omega=0}=0, m=0,1, \ldots, p-1$, then $\psi^{\ell}$ defined by (2.1) has vanishing moments of order $p_{\ell}$, respectively, that is, $\int x^{m} \psi^{\ell} d x=0, m=$ $0,1, \ldots, p_{\ell}-1$. Where $\ell=1,2, \ldots, r$.

\section{Symmetry Transform}

In this section, we will firstly construct a paraunitary matrix based on the polyphase matrix corresponding to 3-band compactly supported wavelet frames. Further, we will establish necessary constraints on the filter lengths and symmetric features of wavelet frames.

\subsection{Construction of Paraunitary Matrix Based on the Polyphase Matrix}

From now on, we only consider the least number wavelet frames with $r=3$ and for the sake of convenience, we rewrit refinement mask and wavelet frame mask associated with $\phi$ and $\psi^{\ell}, \ell=1,2,3$, respectively, that is, $h(z)=\sum_{k \in Z} h_{k} z^{-k}, g(z)=\sum_{k \in Z} g_{k} z^{-k}, f(z)=\sum_{k \in Z} f_{k} z^{-k}$, $s(z)=\sum_{k \in Z} s_{k} z^{-k}$, where $z=e^{i \omega}$.

Suppose casual FIR filter $h, g, f, s$ are symmetric/antisymmetric and $h_{3 k+n}(z)$, $f_{3 k+n}(z), g_{3 k+n}(z), s_{3 k+n}(z), n=0,1,2$ defined by (2.4). For $L \in Z$, define

$$
A_{L}(z)=\left[\begin{array}{llll}
h_{3 k}(z) & h_{3 k+1}(z) & h_{3 k+2}(z) & h_{m}(z) \\
g_{3 k}(z) & g_{3 k+1}(z) & g_{3 k+2}(z) & g_{m}(z) \\
f_{3 k}(z) & f_{3 k+1}(z) & f_{3 k+2}(z) & f_{m}(z) \\
s_{3 k}(z) & s_{3 k+1}(z) & s_{3 k+2}(z) & s_{m}(z)
\end{array}\right]
$$


where

$$
\begin{aligned}
& h_{m}(z)=-z^{-L}\left|\begin{array}{lll}
g_{3 k}\left(z^{-1}\right) & g_{3 k+1}\left(z^{-1}\right) & g_{3 k+2}\left(z^{-1}\right) \\
f_{3 k}\left(z^{-1}\right) & f_{3 k+1}\left(z^{-1}\right) & f_{3 k+2}\left(z^{-1}\right) \\
s_{3 k}\left(z^{-1}\right) & s_{3 k+1}\left(z^{-1}\right) & s_{3 k+2}\left(z^{-1}\right)
\end{array}\right|, \\
& g_{m}(z)=z^{-L}\left|\begin{array}{lll}
h_{3 k}\left(z^{-1}\right) & h_{3 k+1}\left(z^{-1}\right) & h_{3 k+2}\left(z^{-1}\right) \\
f_{3 k}\left(z^{-1}\right) & f_{3 k+1}\left(z^{-1}\right) & f_{3 k+2}\left(z^{-1}\right) \\
s_{3 k}\left(z^{-1}\right) & s_{3 k+1}\left(z^{-1}\right) & s_{3 k+2}\left(z^{-1}\right)
\end{array}\right|, \\
& f_{m}(z)=-z^{-L}\left|\begin{array}{lll}
h_{3 k}\left(z^{-1}\right) & h_{3 k+1}\left(z^{-1}\right) & h_{3 k+2}\left(z^{-1}\right) \\
g_{3 k}\left(z^{-1}\right) & g_{3 k+1}\left(z^{-1}\right) & g_{3 k+2}\left(z^{-1}\right) \\
s_{3 k}\left(z^{-1}\right) & s_{3 k+1}\left(z^{-1}\right) & s_{3 k+2}\left(z^{-1}\right)
\end{array}\right|, \\
& s_{m}(z)=z^{-L}\left|\begin{array}{lll}
h_{3 k}\left(z^{-1}\right) & h_{3 k+1}\left(z^{-1}\right) & h_{3 k+2}\left(z^{-1}\right) \\
g_{3 k}\left(z^{-1}\right) & g_{3 k+1}\left(z^{-1}\right) & g_{3 k+2}\left(z^{-1}\right) \\
f_{3 k}\left(z^{-1}\right) & f_{3 k+1}\left(z^{-1}\right) & f_{3 k+2}\left(z^{-1}\right)
\end{array}\right| .
\end{aligned}
$$

Then $A_{L}(z)$ can be seen as a symmetry paraunitary extension of polyphase matrices $P(z)$ with $r=3$, and have the following theorem and further investigation.

Lemma 3.1. Let $M(z), P(z)$, and $A_{L}(z)$ are defined in (2.4), (2.5), and (3.1). Then the following three statements are equivalent.

(a) $M^{T}(z) M\left(z^{-1}\right)=I_{3}$;

(b) $P^{T}(z) P\left(z^{-1}\right)=I_{3}$;

(c) $A_{L}^{T}(z) A_{L}\left(z^{-1}\right)=I_{4}$.

From the foregoing discussion

$$
\left[\begin{array}{l}
h(z) \\
g(z) \\
f(z) \\
s(z)
\end{array}\right]=\frac{\sqrt{3}}{3} A_{L}\left(z^{3}\right)\left[\begin{array}{c}
1 \\
z^{-1} \\
z^{-2} \\
0
\end{array}\right]
$$

Further, if $h, g, f, s$ are causal, then for a sufficiently large $L, A_{L}$ is also causal. A paraunitary and causal FIR matrix can be factorized into the products of $U_{0} U_{1}(z) \cdots U_{k}(z)[1,3]$, where $k \in N_{0}$. So far, the construction for FIR filters $h, g, f, s$ converted the design for $4 \times 4$ paraunitary matrix $A_{L}(z)$.

\subsection{Investigation on Filter Lengths and Symmetry Features}

The main content of this section is to investigate the constraint conditions of filter lengths and symmetric features of wavelet frames in order to discuss the method for construction framelets with desired properties.

Theorem 3.2. Let $h, g, f, s$ are symmetric/antisymmetric nonzero FIR filters with the centers of symmetry $N / 2, K / 2, S / 2, T / 2$, respectively, where $N, K, S$, and $T \in Z$. If $M$ is paraunitary, then 
(1) leng $(h)=\operatorname{leng}(g)$.

(2) leng $(f)$, leng $(s)$ have the same parity.

Proof. (1) By the conditions of $h, g, f$, s, we have leng $(h)=2 k_{1}+N+1$, leng $(g)=2 k_{2}+K+1$, leng $(f)=2 k_{3}+S+1$, leng $(s)=2 k_{4}+T+1$, where $k_{1}, k_{2}, k_{3}, k_{4} \in Z$. From the paraunitariness of $M$, we have $|h(z)|^{2}+|g(z)|^{2}+|f(z)|^{2}+|s(z)|^{2}=1$, leng $(h)=\operatorname{leng}(g)$. Thus $N-K$ is even.

(2) From the paraunitariness of $M$, we obtain

$$
h(z) h\left(z_{1} z^{-1}\right)+g(z) g\left(z_{1} z^{-1}\right)+f(z) f\left(z_{1} z^{-1}\right)+s(z) s\left(z_{1} z^{-1}\right)=0,
$$

or

$$
h\left(z^{-1}\right) h\left(z_{1} z\right)+g\left(z^{-1}\right) g\left(z_{1} z\right)+f\left(z^{-1}\right) f\left(z_{1} z\right)+s\left(z^{-1}\right) s\left(z_{1} z\right)=0,
$$

where $z_{1}=-1 / 2+(\sqrt{3} / 2) i$ or $z_{1}=-1 / 2-(\sqrt{3} / 2) i$. From the symmetric of $h, g, f, s$, we have

$$
\begin{gathered}
h\left(z^{-1}\right) h\left(z_{1} z\right)=(-1)^{N} h(z) h\left(z_{1} z^{-1}\right), \quad g\left(z^{-1}\right) g\left(z_{1} z\right)=(-1)^{K} g(z) g\left(z_{1} z^{-1}\right), \\
f\left(z^{-1}\right) f\left(z_{1} z\right)=(-1)^{S} f(z) f\left(z_{1} z^{-1}\right), \quad s\left(z^{-1}\right) s\left(z_{1} z\right)=(-1)^{T} s(z) s\left(z_{1} z^{-1}\right) .
\end{gathered}
$$

Further,

$$
(-1)^{N} h(z) h\left(z_{1} z^{-1}\right)+(-1)^{K} g(z) g\left(z_{1} z^{-1}\right)+(-1)^{S} f(z) f\left(z_{1} z^{-1}\right)+(-1)^{T} s(z) s\left(z_{1} z^{-1}\right)=0 .
$$

Thus

$$
\left(1-(-1)^{K-N}\right) g(z) g\left(z_{1} z^{-1}\right)+\left(1-(-1)^{S-N}\right) f(z) f\left(z_{1} z^{-1}\right)+\left(1-(-1)^{T-N}\right) s(z) s\left(z_{1} z^{-1}\right)=0 .
$$

Since $N-K$ is even, hence

$$
\left(1-(-1)^{S-N}\right) f(z) f\left(z_{1} z^{-1}\right)+\left(1-(-1)^{T-N}\right) s(z) s\left(z_{1} z^{-1}\right)=0
$$

Obviously, $T-N$ is even when $S-N$ is even. Assume that $T-N$ is even when $S-N$ is odd, then we have $f(z) f\left(z_{1} z^{-1}\right)=0$, which is contradiction since $f$ is nonzero filter.

Without loss of generality, we give two assumptions of the casual filters $h, g, f, s$ :

(1) leng $(h) \equiv \operatorname{leng}(g) \equiv \operatorname{leng}(f) \equiv \operatorname{leng}(s)(\bmod 3)$;

(2) leng $(h)=\operatorname{leng}(g) \geq \operatorname{leng}(f) \geq \operatorname{leng}(s)$.

Then we have the following Theorem. 
Theorem 3.3. Let

$$
h(z)=\sum_{k=0}^{3 \gamma-1} h_{k} z^{-k}, \quad g(z)=\sum_{k=0}^{3 \gamma-1} g_{k} z^{-k}, \quad f(z)=\sum_{k=0}^{3 m_{1}-1} f_{k} z^{-k}, \quad s(z)=\sum_{k=0}^{3 m_{2}-1} s_{k} z^{-k}
$$

are the causal filters and satisfy $h_{0} \neq 0$ and $\gamma \geq m_{1} \geq m_{2}, \gamma \in N$. Suppose $(3 \gamma-1) / 2$ is the center of $h(z)$ and $g(z)=t_{0} z^{-(3 \gamma-1)} g\left(z^{-1}\right), f(z)=t_{1} z^{-\left(3 m_{1}-1\right)} f\left(z^{-1}\right), s(z)=t_{2} z^{-\left(3 m_{2}-1\right)} s\left(z^{-1}\right), t_{j}= \pm 1, j=$ $0,1,2,3$. If $M$ is a paraunitary matrix, then

(1) leng $\left(h_{3 k}\right)+\gamma$, leng $\left(g_{3 k}\right)+\gamma$, leng $\left(f_{3 k}\right)+m_{1}$, and leng $\left(s_{3 k}\right)+m_{2}$ are even;

(2) $t_{0}+t_{1}+t_{2}+t_{0} t_{1} t_{2}=0,1+t_{0}+(-1)^{\gamma-m_{1}} t_{1}+(-1)^{\gamma-m_{2}} t_{2}+(-1)^{\gamma}\left(t_{0} t_{1} t_{2}-1\right)=0$;

(3) $A_{L}(z)=A_{L_{0}}(z)$ defined by (3.1) with $L_{0}=\gamma+(1 / 2)\left(m_{1}+m_{2}\right)-2$ is causal, paraunitary, and satisfies

$$
A_{L}(z)=z^{-(\gamma-1)} \operatorname{diag}\left(1, t_{0}, t_{1} z^{-\left(m_{1}-\gamma\right)}, t_{2} z^{-\left(m_{2}-\gamma\right)}\right) A_{L}\left(z^{-1}\right) \operatorname{diag}\left(J_{3},-t_{0} t_{1} t_{2}\right)
$$

Proof. By the symmetry of $h, g, f, s$, we have

$$
\begin{array}{cl}
z^{-(\gamma-1)} h_{3 k}\left(z^{-1}\right)=h_{3 k+2}(z), & z^{-(\gamma-1)} h_{3 k+1}\left(z^{-1}\right)=h_{3 k+1}(z) ; \\
z^{-(\gamma-1)} g_{3 k}\left(z^{-1}\right)=t_{0} g_{3 k+2}(z), & z^{-(\gamma-1)} g_{3 k+1}\left(z^{-1}\right)=t_{0} g_{3 k+1}(z) \\
z^{-\left(m_{1}-1\right)} f_{3 k}\left(z^{-1}\right)=t_{1} f_{3 k+2}(z), & z^{-\left(m_{1}-1\right)} f_{3 k+1}\left(z^{-1}\right)=t_{1} f_{3 k+1}(z) \\
z^{-\left(m_{2}-1\right)} s_{3 k}\left(z^{-1}\right)=t_{2} S_{3 k+2}(z), & z^{-\left(m_{2}-1\right)} s_{3 k+1}\left(z^{-1}\right)=t_{2} S_{3 k+1}(z) .
\end{array}
$$

Take them into $h_{m}$ defined by (3.2), we have

$$
\begin{aligned}
h_{m}(z) & =-z^{-L}\left[\begin{array}{lll}
g_{3 k}\left(z^{-1}\right) & g_{3 k+1}\left(z^{-1}\right) & g_{3 k+2}\left(z^{-1}\right) \\
f_{3 k}\left(z^{-1}\right) & f_{3 k+1}\left(z^{-1}\right) & f_{3 k+2}\left(z^{-1}\right) \\
s_{3 k}\left(z^{-1}\right) & s_{3 k+1}\left(z^{-1}\right) & s_{3 k+2}\left(z^{-1}\right)
\end{array}\right] \\
& =-z^{-L}\left[\begin{array}{ccc}
z^{\gamma-1} t_{0} g_{3 k+2}(z) & z^{\gamma-1} t_{0} g_{3 k+1}(z) & z^{\gamma-1} t_{0} g_{3 k}(z) \\
z^{m_{1}-1} t_{1} f_{3 k+2}(z) & z^{m_{1}-1} t_{1} f_{3 k+1}(z) & z^{m_{1}-1} t_{1} f_{3 k}(z) \\
z^{m_{2}-1} t_{2} s_{3 k+2}(z) & z^{m_{2}-1} t_{2} s_{3 k+1}(z) & z^{m_{2}-1} t_{2} s_{3 k}(z)
\end{array}\right] \\
& =z^{-L} \cdot z^{\gamma-1} \cdot z^{m_{1}-1} \cdot z^{m_{1}-1} t_{0} t_{1} t_{2}\left[\begin{array}{ccc}
g_{3 k}(z) & g_{3 k+1}(z) & g_{3 k+2}(z) \\
f_{3 k}(z) & f_{3 k+1}(z) & f_{3 k+2}(z) \\
s_{3 k+2}(z) & s_{3 k+1}(z) & s_{3 k+2}(z)
\end{array}\right] \\
& =-t_{0} t_{1} t_{2} z^{-\left(2 L-\gamma-m_{1}-m_{2}+3\right)} h_{m}\left(z^{-1}\right) .
\end{aligned}
$$


Similarly, for $g_{m}, f_{m}, s_{m}$ defined by (3.3)-(3.5), we also have

$$
\begin{gathered}
g_{m}(z)=-t_{1} t_{2} z^{-\left(2 L-\gamma-m_{1}-m_{2}+3\right)} g_{m}\left(z^{-1}\right), \\
f_{m}(z)=-t_{0} t_{2} z^{-\left(2 L-2 \gamma-m_{2}+3\right)} f_{m}\left(z^{-1}\right), \\
s_{m}(z)=-t_{0} t_{1} z^{-\left(2 L-2 \gamma-m_{1}+3\right)} s_{m}\left(z^{-1}\right) .
\end{gathered}
$$

Thus, $A_{L}(z)$ defined by (3.1) satisfies with

$$
A_{L}(z)=z^{-(\gamma-1)} \operatorname{diag}\left(1, t_{0}, t_{1} z^{-\left(m_{1}-\gamma\right)}, t_{2} z^{-\left(m_{2}-\gamma\right)}\right) A_{L}\left(z^{-1}\right) \operatorname{diag}\left(J_{3},-t_{0} t_{1} t_{2} z^{-\left(2 L-2 \gamma-m_{1}-m_{2}+4\right)}\right) .
$$

From the paraunitariness of $A_{L}(z)$, we obtain leng $\left(h_{m}\right)=\operatorname{leng}\left(h_{3 k}\right)$. Assume that

$$
h_{m}(z)=a_{l} z^{-k}+a_{l+1} z^{-(l+1)}+\cdots+\left(-t_{0} t_{1} t_{2}\right) z^{-\left(l+\operatorname{leng}\left(h_{3 k}\right)-1\right)}, \quad l \in Z, a_{l} \neq 0
$$

By symmetric of $h$, we have $z^{-\left(l+\text { leng }\left(h_{3 k}\right)-1\right)} h_{m}\left(z^{-1}\right)=-t_{0} t_{1} t_{2} h_{m}(z)$, which together with (3.17) leads to $2 l+\operatorname{leng}\left(h_{3 k}\right)-1=2 L-\gamma-m_{1}-m_{2}+3$; thus, leng $\left(h_{3 k}\right)+\gamma+m_{1}+m_{2}=2 L-2 l+4$ is even. Note that $3 m_{1}$ and $3 m_{2}$ have the same parity by Theorem 3.2, hence $m_{1}+m_{2}$ is even, leng $\left(h_{3 k}\right)+\gamma$ is even. One can prove similarly that leng $\left(g_{3 k}\right)+\gamma$, leng $\left(f_{3 k}\right)+m_{1}$ and leng $\left(s_{3 k}\right)+$ $m_{2}$ are all even. satisfy

Let $L_{0}=\gamma+(1 / 2)\left(m_{1}+m_{2}\right)-2$. Then $h_{m}, g_{m}, f_{m}, s_{m}$ defined by (3.2)-(3.5) with $L_{0}$

$$
\begin{aligned}
& h_{m}(z)=-t_{0} t_{1} t_{2} z^{-(\gamma-1)} h_{m}\left(z^{-1}\right), \quad g_{m}(z)=-t_{1} t_{2} z^{-(\gamma-1)} g_{m}\left(z^{-1}\right), \\
& f_{m}(z)=-t_{0} t_{2} z^{-\left(m_{1}-1\right)} f_{m}\left(z^{-1}\right), \quad s_{m}(z)=-t_{0} t_{1} z^{-\left(m_{2}-1\right)} s_{m}\left(z^{-1}\right) .
\end{aligned}
$$

Note that

$$
\begin{aligned}
& \operatorname{leng}\left(h_{m}\right)=\operatorname{leng}\left(h_{3 k}\right) \leq \gamma=\gamma-1+1, \quad \operatorname{leng}\left(g_{m}\right)=\operatorname{leng}\left(g_{3 k}\right) \leq r=r-1+1, \\
& \operatorname{leng}\left(f_{m}\right)=\operatorname{leng}\left(f_{3 k}\right) \leq m_{1}=m_{1}-1+1, \quad \operatorname{leng}\left(s_{m}\right)=\operatorname{leng}\left(s_{3 k}\right) \leq m_{2} .
\end{aligned}
$$

Thus, $h_{m}, g_{m}, f_{m}, s_{m}$ are casual, $A(z)=A_{L_{0}}(z)$ is casual, paraunitary, and satisfies (3.14).

Set $z=1$ and $z=-1$ in (3.14), then we have

$$
\begin{gathered}
\operatorname{Tr}\left(\operatorname{diag}\left(1, t_{0}, t_{1}, t_{2}\right)\right)=\operatorname{Tr}\left(\operatorname{diag}\left(J_{3},-t_{0} t_{1} t_{2}\right)\right), \\
\operatorname{Tr}\left((-1)^{-\gamma} \operatorname{diag}\left(1, t_{0},(-1)^{\gamma-m_{1}} t_{1},(-1)^{\gamma-m_{2}} t_{2}\right)\right)=\operatorname{Tr}\left(\operatorname{diag}\left(J_{3},-t_{0} t_{1} t_{2}\right)\right),
\end{gathered}
$$

which imply (2.2). 
With similar arguments we can prove the others case and present the following two theorems as follows.

Theorem 3.4. Let

$$
h(z)=\sum_{k=0}^{3 \gamma} h_{k} z^{-k}, \quad g(z)=\sum_{k=0}^{3 \gamma} g_{k} z^{-k}, \quad f(z)=\sum_{k=0}^{3 m_{1}} f_{k} z^{-k}, \quad s(z)=\sum_{k=0}^{3 m_{2}} s_{k} z^{-k},
$$

are the causal filters and satisfy $h_{0} \neq 0$ and $\gamma \geq m_{1} \geq m_{2}, \gamma \in N$. Suppose $(3 \gamma) / 2$ is the center of $h(z)$ and $g(z)=t_{0} z^{-3 \gamma} g\left(z^{-1}\right), f(z)=t_{1} z^{-3 m_{1}} f\left(z^{-1}\right), s(z)=t_{2} z^{-3 m_{2}} s\left(z^{-1}\right), t_{j}= \pm 1, j=0,1,2,3$. If $M$ is a paraunitary matrix, then

(1) $\gamma+m_{1}$ and $\gamma+m_{2}$ are even.

(2) $t_{0}+t_{1}+t_{2}+t_{0} t_{1} t_{2}=0,1+t_{0}+(-1)^{\gamma-m_{1}} t_{1}+(-1)^{\gamma-m_{2}} t_{2}+(-1)^{\gamma}\left(t_{0} t_{1} t_{2}-1\right)=0$.

(3) $A_{L}(z)=A_{L_{0}}(z)$ defined by (3.1) with $L_{0}=\gamma+(1 / 2)\left(m_{1}+m_{2}-2\right)$ is causal, paraunitary and satisfies

$$
A_{L}(z)=z^{-\gamma} \operatorname{diag}\left(1, t_{0}, t_{1} z^{-\left(m_{1}-\gamma\right)}, t_{2} z^{-\left(m_{2}-\gamma\right)}\right) A_{L}\left(z^{-1}\right) \operatorname{diag}\left(1, z J_{2},-t_{0} t_{1} t_{2}\right)
$$

Theorem 3.5. Let

$$
h(z)=\sum_{k=0}^{3 \gamma+1} h_{k} z^{-k}, \quad g(z)=\sum_{k=0}^{3 \gamma+1} g_{k} z^{-k}, \quad f(z)=\sum_{k=0}^{3 m_{1}+1} f_{k} z^{-k}, \quad s(z)=\sum_{k=0}^{3 m_{2}+1} s_{k} z^{-k}
$$

are the causal filters and satisfy $h_{0} \neq 0$ and $\gamma \geq m_{1} \geq m_{2}, \gamma \in N$. Suppose $(3 \gamma+1) / 2$ is the center of $h(z)$ and $g(z)=t_{0} z^{-(3 \gamma+1)} g\left(z^{-1}\right), f(z)=t_{1} z^{-\left(3 m_{1}+1\right)} f\left(z^{-1}\right), s(z)=t_{2} z^{-\left(3 m_{2}+1\right)} s\left(z^{-1}\right), t_{j}= \pm 1$, $j=0,1,2,3$. If $M$ is a paraunitary matrix, then

(1) leng $\left(h_{3 k}\right)+r$, leng $\left(g_{3 k}\right)+\gamma$, leng $\left(f_{3 k}\right)+m_{1}$, and leng $\left(s_{3 k}\right)+m_{2}$ are even;

(2) $t_{0}+t_{1}+t_{2}+t_{0} t_{1} t_{2}=0,1+t_{0}+(-1)^{\gamma-m_{1}} t_{1}+(-1)^{\gamma-m_{2}} t_{2}+(-1)^{\gamma}\left(t_{0} t_{1} t_{2}-1\right)=0$;

(3) $A_{L}(z)=A_{L_{0}}(z)$ defined by (3.1) with $L_{0}=\gamma+(1 / 2)\left(m_{1}+m_{2}\right)$ is causal, paraunitary and satisfies

$$
A_{L}(z)=z^{-(\gamma+1)} \operatorname{diag}\left(1, t_{0}, t_{1} z^{-\left(m_{1}-\gamma\right)}, t_{2} z^{-\left(m_{2}-\gamma\right)}\right) A_{L}\left(z^{-1}\right) \operatorname{diag}\left(J_{2}, z,-t_{0} t_{1} t_{2}\right)
$$

So far, we have completed the further extension of the our previous work in [22], that is, we presented the several necessary constraints on the filter lengths and symmetric features of wavelet frames. By using these properties, $A_{L}(z)$ defined by (3.1) can be divided into several categories according to the different length of masks. Then we can improve the condition of $A_{L}(z)$ in the following work and prepare for the parameterizations. This paper is devoted to give the parameterizations of 3-band tight wavelet frames with $3 N+1$ filter lengths. 


\section{The Parameterizations for Combined MRA Masks with $3 N+1$ Filter Lengths}

For simplicity we give the following two assumptions:

(1) leng $(h)=$ leng $(g)=3 \gamma+1$, leng $(f)=$ leng $(s)=3 m+1$;

(2) $m \equiv 1(\bmod 3), 0 \leq \gamma-m \leq 2$.

\subsection{The Case of $\gamma=3 n+1$}

For $A_{L}(z)$ with $L=6 n+1$ in (3.1), denote $A(z)=A_{L}(z)$, then (3.24) can be converted to

$$
A(z)=z^{-(3 n+1)} \operatorname{diag}\left(1, t_{0}, t_{1}, t_{2}\right) A\left(z^{-1}\right) \operatorname{diag}\left(1, \operatorname{diag}(z, z) J_{2},-t_{0} t_{1} t_{2}\right) .
$$

While

$$
t_{0}+t_{1}+t_{2}+t_{0} t_{1} t_{2}=0, \quad t_{i}= \pm 1, \quad i=1,2,3
$$

imply that

(i) The first case: one of $t_{i}$ is 1 and the other two are -1 ;

(ii) The second case: one of $t_{i}$ is -1 and the other two are 1 .

Lemma 4.1. For $A(z)$ in (4.1), let

$$
B(z)=A(z) \operatorname{diag}\left(1, R_{1}, 1\right),
$$

where $R_{1}=(\sqrt{2} / 2)\left[\begin{array}{cc}1 & -1 \\ 1 & 1\end{array}\right]$, then $A(z)$ satisfies if and only if $B(z)$ satisfies

$$
B(z)=z^{-\gamma} \operatorname{diag}\left(1, t_{0}, t_{1}, t_{2}\right) B\left(z^{-1}\right) \operatorname{diag}\left(1, z,-z,-t_{0} t_{1} t_{2}\right) .
$$

(i) For the first case, without loss of generality, suppose $t_{0}=1, t_{1}=t_{2}=-1$, (4.4) is converted to

$$
z^{-(3 n+1)} \operatorname{diag}(1,1,-1,-1) B\left(z^{-1}\right) \operatorname{diag}(1, z,-z,-1)=B(z) .
$$


Journal of Applied Mathematics

To give the parameterizations of $B(z)$ satisfying (4.5), one defines

$$
W_{0}(z)=\left[\begin{array}{cccc}
-\frac{\sqrt{2}}{4} & -\frac{\sqrt{2}}{4} & \frac{\sqrt{2}}{4} & \frac{\sqrt{2}}{4} \\
\frac{\sqrt{2}}{2} & -\frac{\sqrt{2}}{2} & 0 & 0 \\
0 & 0 & \frac{\sqrt{2}}{2} & -\frac{\sqrt{2}}{2} \\
-\frac{\sqrt{2}}{4} & -\frac{\sqrt{2}}{4} & \frac{\sqrt{2}}{4} & \frac{\sqrt{2}}{4}
\end{array}\right]+\left[\begin{array}{cccc}
-\frac{\sqrt{2}}{4} & -\frac{\sqrt{2}}{4} & -\frac{\sqrt{2}}{4} & -\frac{\sqrt{2}}{4} \\
0 & 0 & 0 & 0 \\
0 & 0 & 0 & 0 \\
\frac{\sqrt{2}}{4} & \frac{\sqrt{2}}{4} & \frac{\sqrt{2}}{4} & \frac{\sqrt{2}}{4}
\end{array}\right] z .
$$

One can check that $W_{0}(z)$ is paraunitary and satisfies

$$
W_{0}(z)=z \operatorname{diag}\left(1, z^{-1},-z^{-1},-1\right) W_{0}\left(z^{-1}\right) \operatorname{diag}(1,1,-1,-1) \text {. }
$$

Lemma 4.2. For $B(z)$ in (4.3), define

$$
E(z)=B(z) W_{0}(z)
$$

Then $E(z)$ satisfies

$$
E(z)=z^{-3 n} \operatorname{diag}(1,1,-1,-1) E\left(z^{-1}\right) \operatorname{diag}(1,1,-1,-1) .
$$

Lemma 4.3. Let a paraunitary matrix $E(z)$ defined by (4.8), then for $n=0, E(z)$ have the form of

$$
P_{0}=\left[\begin{array}{cccc}
\cos \theta_{1} & \rho_{1} \sin \theta_{1} & 0 & 0 \\
\sin \theta_{1} & -\rho_{1} \cos \theta_{1} & 0 & 0 \\
0 & 0 & \cos \theta_{2} & \rho_{2} \sin \theta_{2} \\
0 & 0 & \sin \theta_{2} & -\rho_{2} \cos \theta_{2}
\end{array}\right],
$$

where $\theta \in[-\pi, \pi), \rho_{j}= \pm 1, j \in Z$.

Proof. Let $n=0,(4.9)$ is converted to

$$
E(z)=\operatorname{diag}(1,1,-1,-1) E(z) \operatorname{diag}(1,1,-1,-1) .
$$

If set $4 \times 4$ matrix $P_{0}=\left[a_{i j}\right], i, j=1,2,3,4$, then we can obtain

$$
\begin{array}{ccc}
a_{11}^{2}+a_{12}^{2}=1, & a_{21}^{2}+a_{22}^{2}=1, & a_{11} a_{21}+a_{12} a_{22}=0, \\
a_{33}^{2}+a_{34}^{2}=1, & a_{43}^{2}+a_{44}^{2}=1, & a_{33} a_{43}+a_{34} a_{44}=0, \\
a_{13}=a_{14}=a_{23}=a_{24}=a_{31}=a_{32}=a_{41}=a_{42}=0 .
\end{array}
$$

Assume that $a_{11}=\cos \theta_{1}, a_{33}=\cos \theta_{2}$, then (4.10) is obtained immediately. 
Remark 4.4. For convenience, we only choose the following form of $P_{0}$ in the following:

$$
P_{0}=\left[\begin{array}{cccc}
\cos \theta & \sin \theta & 0 & 0 \\
\sin \theta & -\cos \theta & 0 & 0 \\
0 & 0 & \cos \theta & \sin \theta \\
0 & 0 & \sin \theta & -\cos \theta
\end{array}\right]
$$

Lemma 4.5. Let a paraunitary matrix $E(z)$ satisfies (4.8), then for $n=1, E(z)$ have the form of

$$
\begin{aligned}
V(z)= & \frac{1}{2}\left[\begin{array}{l}
0 \\
1 \\
1 \\
0
\end{array}\right]\left[\cos \alpha_{1}, \sin \alpha_{1}, \cos \alpha_{2}, \sin \alpha_{2}\right] \\
& +\frac{1}{2}\left[\begin{array}{l}
1 \\
0 \\
0 \\
1
\end{array}\right]\left[\sin \alpha_{1},-\cos \alpha_{1}, \sin \alpha_{2},-\cos \alpha_{2}\right] z^{-1} \\
& +\frac{1}{2}\left[\begin{array}{c}
1 \\
0 \\
0 \\
-1
\end{array}\right]\left[\sin \alpha_{1},-\cos \alpha_{1},-\sin \alpha_{2}, \cos \alpha_{2}\right] z^{-2} \\
& +\frac{1}{2}\left[\begin{array}{c}
0 \\
1 \\
-1 \\
0
\end{array}\right]\left[\cos \alpha_{1}, \sin \alpha_{1},-\cos \alpha_{2},-\sin \alpha_{2}\right] z^{-3},
\end{aligned}
$$

where $\alpha_{j} \in[-\pi, \pi), j=1,2$.

Proof. When $n=1$, (4.9) is converted to

$$
E(z)=z^{-3} \operatorname{diag}(1,1,-1,-1) E\left(z^{-1}\right) \operatorname{diag}(1,1,-1,-1) .
$$

Suppose $E(z)=A+B z^{-1}+C z^{-2}+D z^{-3}$, here $A, B, C, D$ are all $4 \times 4$ matrices. Let $A=\left[a_{i j}\right]$ then, from the paraunitariness of $E(z)$, we have

$$
\begin{gathered}
A=\frac{1}{2}\left[\begin{array}{l}
a \\
b \\
c \\
d
\end{array}\right]\left[\cos \alpha_{1}, \sin \alpha_{1}, \cos \alpha_{2}, \sin \alpha_{2}\right], \\
D=\frac{1}{2}\left[\begin{array}{l}
e \\
f \\
g \\
h
\end{array}\right]\left[\cos \alpha_{1}, \sin \alpha_{1},-\cos \alpha_{2},-\sin \alpha_{2}\right],
\end{gathered}
$$


where $b, c, f, g$ are all not zeros or $a, d, e, h$ are all not zeros. Now suppose $b, c, f, g$ are all not zeros, then $b=f=1, c=-g=1$.

Similarly, $B$ and $C$ are given as follows:

$$
\begin{aligned}
& B=\frac{1}{2}\left[\begin{array}{l}
1 \\
0 \\
0 \\
1
\end{array}\right]\left[\sin \alpha_{1},-\cos \alpha_{1}, \sin \alpha_{2},-\cos \alpha_{2}\right] \\
& C=\frac{1}{2}\left[\begin{array}{l}
a \\
b \\
c \\
d
\end{array}\right]\left[\sin \alpha_{1},-\cos \alpha_{1},-\sin \alpha_{2}, \cos \alpha_{2}\right]
\end{aligned}
$$

Equation (4.14) is obtained.

Theorem 4.6. A causal paraunitary matrix filter $E(z)$ defined by (4.8) for some $n \in N$ if and only if it can be factorized in the form of

$$
E(z)=P_{0} V_{1}(z) \cdots V_{n}(z),
$$

where $P_{0}$ is defined by (4.13) with $\theta \in[-\pi, \pi)$ and $V_{j}(z), j=1,2$ are defined by (4.14) with $\alpha_{j} \in[-\pi, \pi), j=1,2$.

Proof. If $E(z)$ can be factorized as (4.18), then it is a causal paraunitary filter and satisfies (4.9). Conversely, we set a paraunitary matrix $E_{n}(z)=e_{0}+e_{1} z^{-1}+\cdots+e_{n} z^{-3 n}$ satisfies with (4.18), where $n \in N$. If $n=0$, it is easy to get that $E(z)$ is $P_{0}$. For $n \in N \backslash\{0\}$, define

$$
E_{n-1}(z)=E_{n}(z) V^{T}\left(z^{-1}\right)
$$

where $V(z)$ is defined by (4.14). Now we prove that there exists $\alpha_{1}, \alpha_{2}$ such that $E_{n-1}(z)$ defined by (4.19) is causal. From $V^{T}\left(z^{-1}\right)$, we only need to prove that there exists $\alpha_{1}, \alpha_{2}$ such that

$$
\begin{aligned}
& e_{0}\left[\sin \alpha_{1},-\cos \alpha_{1}, \sin \alpha_{2},-\cos \alpha_{2}\right]^{T}=0, \\
& e_{0}\left[\sin \alpha_{1},-\cos \alpha_{1},-\sin \alpha_{2}, \cos \alpha_{2}\right]^{T}=0, \\
& e_{0}\left[\cos \alpha_{1}, \sin \alpha_{1},-\cos \alpha_{2},-\sin \alpha_{2}\right]^{T}=0, \\
& e_{1}\left[\sin \alpha_{1},-\cos \alpha_{1},-\sin \alpha_{2}, \cos \alpha_{2}\right]^{T}=0, \\
& e_{1}\left[\cos \alpha_{1}, \sin \alpha_{1},-\cos \alpha_{2},-\sin \alpha_{2}\right]^{T}=0, \\
& e_{2}\left[\cos \alpha_{1}, \sin \alpha_{1},-\cos \alpha_{2},-\sin \alpha_{2}\right]^{T}=0 .
\end{aligned}
$$

Let us start by discussing the case $e_{0}=0$. Then (4.20)-(4.22) hold. By the symmetry of $E_{n}(z), \quad e_{j}=\operatorname{diag}(1,1,-1,-1) e_{3 n-j} \operatorname{diag}(1,1,-1,-1), 0 \leq j \leq 3 n$. By the paraunitariness of 
$E_{n}(z), e_{1} e_{3 n-1}^{T}=0$. Then we have $e_{1} \operatorname{diag}(1,1,-1,-1) e_{1}^{T}=0, \operatorname{rank}\left(e_{1}\right) \leq 2$. For simplicity, we only consider the case $\operatorname{rank}\left(e_{1}\right)=1$ in the following. Let $e_{1}=u_{1}\left[\cos \theta_{1}, \sin \theta_{1}, \cos \theta_{2}, \sin \theta_{2}\right]$, $u_{1} \in R^{4}, \theta_{1}, \theta_{2} \in[-\pi, \pi)$. Then for $\alpha_{1}=\theta_{1}, \alpha_{2}=\theta_{2}$, (4.23) and (4.24) hold. Let $Q=e_{2}$ $\left[\cos \theta_{1}, \sin \theta_{1},-\cos \theta_{2},-\sin \theta_{2}\right]^{T}$, by the paraunitariness of $E_{n}(z), e_{1} e_{3 n-1}^{T}+e_{2} e_{3 n}^{T}=0$. Then we have $u_{1} Q^{T}+Q u_{1}^{T}=0$. It is clear that $Q=0$ since $u_{1} \neq 0$. Then (4.25) holds for $\alpha_{1}=\theta_{1}, \alpha_{2}=\theta_{2}$.

If $e_{0} \neq 0$, we have $e_{0} e_{3 n}^{T}=0$ and $e_{3 n}=\operatorname{diag}(1,1,-1,-1) e_{0} \operatorname{diag}(1,1,-1,-1)$. Let $e_{0}=u_{1}$ [ $\cos \theta_{1}, \sin \theta_{1}, \cos \theta_{2}, \sin \theta_{2}$, then (4.20)-(4.22) hold for $\alpha_{1}=\theta_{1}, \alpha_{2}=\theta_{2}$. Similarly, when $e_{1}=u_{2}\left[-\sin \theta_{1}, \cos \theta_{1},-\sin \theta_{2}, \cos \theta_{2}\right],(4.23)$, and (4.24) hold for $\alpha_{1}=\theta_{1}, \alpha_{2}=\theta_{2}$. Let $Q=e_{2}\left[\cos \alpha_{1}, \sin \alpha_{1},-\cos \alpha_{2},-\sin \alpha_{2}\right]^{T}$, from the paraunitariness of $E_{n}(z)$, we have $e_{1} e_{3 n-1}^{T}+$ $e_{2} e_{3 n}^{T}=0$. Thus, $u_{2} Q^{T}+Q u_{2}^{T}=0$. It is clear that $Q=0$ since $u_{2} \neq 0$. So (4.25) holds for $\alpha_{1}=\theta_{1}$, $\alpha_{2}=\theta_{2}$.

So $E_{n-1}(z)$ is causal and $E_{n-1}(z)$ can be factorized in the form of $E_{n-1}(z)=P_{0} V_{1}(z) \cdots$ $V_{n-1}(z)$. Thus, $E_{n}(z)$ can be factorized in the form of $E_{n}(z)=P_{0} V_{1}(z) \cdots V_{n-1}(z)$ by induction assumption. The proof of Theorem 4.6 is complete.

Therefore, we have the following corollary.

Corollary 4.7. Let $h(z), g(z), f(z), s(z)$ is symmetric or antisymmetric. If $M$ is a paraunitary matrix, then $h(z), g(z), f(z), s(z)$ can be factorized as

$$
\left[\begin{array}{l}
h(z) \\
g(z) \\
f(z) \\
s(z)
\end{array}\right]=\frac{\sqrt{6}}{12} P_{0} V_{1}\left(z^{3}\right) \cdots V_{n}\left(z^{3}\right)\left[\begin{array}{c}
-1+\sqrt{2} z^{-1}+\sqrt{2} z^{-2}-z^{-3} \\
-1-\sqrt{2} z^{-1}-\sqrt{2} z^{-2}-z^{-3} \\
1-\sqrt{2} z^{-1}+\sqrt{2} z^{-2}-z^{-3} \\
1+\sqrt{2} z^{-1}-\sqrt{2} z^{-2}-z^{-3}
\end{array}\right]
$$

for the case of $t_{0}=1, t_{1}=t_{2}=-1$;

$$
\left[\begin{array}{l}
h(z) \\
g(z) \\
f(z) \\
s(z)
\end{array}\right]=\frac{\sqrt{6}}{12} V_{0} V_{1}\left(z^{3}\right) \cdots V_{n}\left(z^{3}\right)\left[\begin{array}{c}
-1+\sqrt{2} z^{-1}+\sqrt{2} z^{-2}-z^{-3} \\
-1-\sqrt{2} z^{-1}-\sqrt{2} z^{-2}-z^{-3} \\
1-\sqrt{2} z^{-1}+\sqrt{2} z^{-2}-z^{-3} \\
1+\sqrt{2} z^{-1}-\sqrt{2} z^{-2}-z^{-3}
\end{array}\right]
$$

for the case of $t_{0}=-1, t_{1}=1, t_{2}=-1$, where $V_{0}=\operatorname{diag}\left(1, J_{2}, 1\right) P_{0}$;

$$
\left[\begin{array}{l}
h(z) \\
g(z) \\
f(z) \\
s(z)
\end{array}\right]=\frac{\sqrt{6}}{12} U_{0} V_{1}\left(z^{3}\right) \cdots V_{n}\left(z^{3}\right)\left[\begin{array}{c}
-1+\sqrt{2} z^{-1}+\sqrt{2} z^{-2}-z^{-3} \\
-1-\sqrt{2} z^{-1}-\sqrt{2} z^{-2}-z^{-3} \\
1-\sqrt{2} z^{-1}+\sqrt{2} z^{-2}-z^{-3} \\
1+\sqrt{2} z^{-1}-\sqrt{2} z^{-2}-z^{-3}
\end{array}\right]
$$

for the case of $t_{0}=t_{1}=-1, t_{2}=1$, where $U_{0}=\operatorname{diag}\left(1, J_{3}\right) P_{0}$.

Where $P_{0}$ is defined by (4.13) with $\theta \in[-\pi, \pi)$ and $V_{j}(z)$ are defined by (4.14) with $\alpha_{j} \in$ $[-\pi, \pi)$. 
(ii) For the second case, without loss of generality, suppose $t_{0}=1, t_{1}=1, t_{2}=-1$, then $E(z)$ defined by (4.8) satisfies

$$
E(z)=z^{-3 n} \operatorname{diag}(1,1,1,-1) E\left(z^{-1}\right) \operatorname{diag}(1,1,-1,1) .
$$

Let $n=0$ in (4.29), then one can get an orthogonal matrix $Q_{0}$ as follows:

$$
Q_{0}=\left[\begin{array}{cccc}
\cos \theta & 0 & 0 & -\rho_{1} \sin \theta \\
0 & \rho_{2} & 0 & 0 \\
0 & 0 & \rho_{3} & 0 \\
\sin \theta & 0 & 0 & \rho_{1} \cos \theta
\end{array}\right]
$$

Then one has the following results.

Theorem 4.8. Let $E(z)$ is a causal paraunitary matrix filter and satisfies (4.29) for some $n \in N$, then $E(z)$ cannot be factorized in the form of

$$
E(z)=Q_{0} V_{1}(z) \cdots V_{n}(z)
$$

for $Q_{0}$ defined by (4.30) with $\theta \in[-\pi, \pi)$ and $V_{j}(z), j=1,2$ defined by (4.14) with $\alpha_{j} \in[-\pi, \pi)$, $j=1,2$.

Proof. Suppose $E(z)$ can be factorized in the form of (4.31). Then when $n=1$, we have

$$
V_{1}(z)=z^{-3} \operatorname{diag}(1,1,1,-1) V_{1}\left(z^{-1}\right) \operatorname{diag}(1,1,-1,1)
$$

when $n=2$, we have

$$
V_{2}(z)=z^{-3} \operatorname{diag}(1,1,-1,1) V_{2}\left(z^{-1}\right) \operatorname{diag}(1,1,-1,1)
$$

However, (4.33) is in contradiction with (4.32).

\subsection{The Case of $\gamma=3 n+2$}

We have the following results.

Theorem 4.9. $E(z)$ is a causal paraunitary matrix filter defined by (4.8) for some $n \in N$ if and only if it can be factorized in the form of

$$
E(z)=P_{1} V_{2}(z) V_{3}(z) \cdots V_{n}(z),
$$


where $P_{1}$ is defined by the following with $\theta_{j} \in[-\pi, \pi), j=1,2$

$$
\begin{aligned}
P_{1}(z)= & \frac{1}{2}\left[\begin{array}{cccc}
\cos \theta_{1} & \sin \theta_{1} & \cos \theta_{2} & \sin \theta_{2} \\
\cos \theta_{1} & \sin \theta_{1} & \cos \theta_{2} & \sin \theta_{2} \\
-2 \sin \theta_{1} & 2 \cos \theta_{1} & 0 & 0 \\
0 & 0 & -2 \sin \theta_{2} & 2 \cos \theta_{2}
\end{array}\right] \\
& +\frac{1}{2}\left[\begin{array}{cccc}
\cos \theta_{1} & \sin \theta_{1} & -\cos \theta_{2} & -\sin \theta_{2} \\
-\cos \theta_{1} & -\sin \theta_{1} & \cos \theta_{2} & \sin \theta_{2} \\
0 & 0 & 0 & 0 \\
0 & 0 & 0 & 0
\end{array}\right] z^{-1},
\end{aligned}
$$

and $V_{j}(z)$ are defined by (4.14) with $\alpha_{j} \in[-\pi, \pi)$.

Corollary 4.10. Let $h(z), g(z), f(z), s(z)$ is symmetric or antisymmetric. If $M$ is a paraunitary matrix, then $h(z), g(z), f(z), s(z)$ can be factorized as

$$
\left[\begin{array}{l}
h(z) \\
g(z) \\
f(z) \\
s(z)
\end{array}\right]=\frac{\sqrt{6}}{12} P_{1}(z) V_{2}\left(z^{3}\right) \cdots V_{n}\left(z^{3}\right)\left[\begin{array}{c}
-1+\sqrt{2} z^{-1}+\sqrt{2} z^{-2}-z^{-3} \\
-1-\sqrt{2} z^{-1}-\sqrt{2} z^{-2}-z^{-3} \\
1-\sqrt{2} z^{-1}+\sqrt{2} z^{-2}-z^{-3} \\
1+\sqrt{2} z^{-1}-\sqrt{2} z^{-2}-z^{-3}
\end{array}\right]
$$

for the case $t_{0}=-1, t_{1}=1, t_{2}=-1$;

$$
\left[\begin{array}{l}
h(z) \\
g(z) \\
f(z) \\
s(z)
\end{array}\right]=\frac{\sqrt{6}}{12} \operatorname{diag}\left(1,1, J_{2}\right) P_{1}(z) V_{2}\left(z^{3}\right) \cdots V_{n}\left(z^{3}\right)\left[\begin{array}{c}
-1+\sqrt{2} z^{-1}+\sqrt{2} z^{-2}-z^{-3} \\
-1-\sqrt{2} z^{-1}-\sqrt{2} z^{-2}-z^{-3} \\
1-\sqrt{2} z^{-1}+\sqrt{2} z^{-2}-z^{-3} \\
1+\sqrt{2} z^{-1}-\sqrt{2} z^{-2}-z^{-3}
\end{array}\right]
$$

for the case of $t_{0}=t_{1}=-1, t_{2}=1$. Where $P_{1}(z)$ is defined by (4.35) with $\theta \in[-\pi, \pi)$ and $V_{j}(z)$ are defined by (4.14) with $\alpha_{j} \in[-\pi, \pi)$.

Remark 4.11. In the case of $t_{0}=1, t_{1}=t_{2}=-1$, we cannot construct a paraunitary matrix $P_{1}(z)$. The reason is similarly as Theorem 4.8 when $t_{0}=1, t_{1}=t_{2}=-1$.

\subsection{The Case of $\gamma=3 n+3$}

In this situation, we cannot also construct the corresponding paraunitary matrix.

\section{Examples}

In this section, we will construction some examples of 3-band wavelet frames with symmetry/antisymmetry by applying the parameterizations of masks $h, g, f, s$ provided in Corollarys 4.7 and 4.10 . 
Example 5.1. Let $h, g, f, s$ be the filters given by (4.36) with $n=1$. Then

$$
\begin{aligned}
& h(z)=\frac{\sqrt{6}}{24}\left[\left(-\cos \theta_{1}-\sin \theta_{1}+\cos \theta_{2}+\sin \theta_{2}\right)+\sqrt{2}\left(\cos \theta_{1}-\sin \theta_{1}-\cos \theta_{2}+\sin \theta_{2}\right) z^{-1}\right. \\
& +\sqrt{2}\left(\cos \theta_{1}-\sin \theta_{1}+\cos \theta_{2}-\sin \theta_{2}\right) z^{-2}-2\left(\cos \theta_{1}+\sin \theta_{1}+\cos \theta_{2}+\sin \theta_{2}\right) z^{-3} \\
& +\sqrt{2}\left(\cos \theta_{1}-\sin \theta_{1}+\cos \theta_{2}-\sin \theta_{2}\right) z^{-4}+\sqrt{2}\left(\cos \theta_{1}-\sin \theta_{1}-\cos \theta_{2}+\sin \theta_{2}\right) z^{-5} \\
& \left.+\left(-\cos \theta_{1}-\sin \theta_{1}+\cos \theta_{2}+\sin \theta_{2}\right) z^{-6}\right], \\
& g(z)=\frac{\sqrt{6}}{24}\left[\left(-\cos \theta_{1}-\sin \theta_{1}+\cos \theta_{2}+\sin \theta_{2}\right)+\sqrt{2}\left(\cos \theta_{1}-\sin \theta_{1}-\cos \theta_{2}+\sin \theta_{2}\right) z^{-1}\right. \\
& +\sqrt{2}\left(\cos \theta_{1}-\sin \theta_{1}+\cos \theta_{2}-\sin \theta_{2}\right) z^{-2}-\sqrt{2}\left(\cos \theta_{1}-\sin \theta_{1}+\cos \theta_{2}-\sin \theta_{2}\right) z^{-4} \\
& \left.+\sqrt{2}\left(\cos \theta_{1}-\sin \theta_{1}-\cos \theta_{2}+\sin \theta_{2}\right) z^{-5}-\left(-\cos \theta_{1}-\sin \theta_{1}+\cos \theta_{2}+\sin \theta_{2}\right) z^{-6}\right], \\
& f(z)=\frac{\sqrt{6}}{24}\left[\left(2 \sin \theta_{1}-2 \cos \theta_{1}\right)+\sqrt{2}\left(-2 \sin \theta_{1}-2 \cos \theta_{1}\right) z^{-1}+\sqrt{2}\left(-2 \sin \theta_{1}-2 \cos \theta_{1}\right) z^{-2}\right] \\
& +\left(2 \sin \theta_{1}-2 \cos \theta_{1}\right) z^{-3}, \\
& s(z)=\frac{\sqrt{6}}{24}\left[\left(-2 \sin \theta_{1}+2 \cos \theta_{1}\right)+\sqrt{2}\left(2 \sin \theta_{1}+2 \cos \theta_{1}\right) z^{-1}+\sqrt{2}\left(-2 \sin \theta_{1}-2 \cos \theta_{1}\right) z^{-2}\right] \\
& +\left(2 \sin \theta_{1}-2 \cos \theta_{1}\right) z^{-3} \text {. }
\end{aligned}
$$

For $h(1)=1, g(1)=f(1)=s(1)=0, h(k)=0, k=-1 / 2 \pm(\sqrt{3} / 2) i$, we have $\cos \theta_{1}=$ $-\sqrt{3} / 6(\sqrt{2}-2), \sin \theta_{1}=-\sqrt{3} / 6(2+\sqrt{2}), \cos \theta_{2}=\sigma, \sin \theta_{2}= \pm \sqrt{1-\sigma^{2}}$. Let $h$ has sum rules of order 2 , such as $h^{\prime}(k)=0$, then we can get that $\sin \theta_{2}=-\sqrt{3} / 18(2+5 \sqrt{2}), \cos \theta_{2}=-\sqrt{3} / 18(-2+$ $5 \sqrt{2})$. Thus

$$
\begin{array}{ll}
h_{0}=-\frac{1}{18}, \quad h_{1}=\frac{1}{9}, \quad h_{2}=\frac{2}{9}, \quad h_{3}=\frac{4}{9}, \quad h_{4}=h_{2}, \quad h_{5}=h_{1}, \quad h_{6}=h_{0}, \\
g_{0}=-\frac{1}{18}, \quad g_{1}=\frac{1}{9}, \quad g_{2}=\frac{2}{9}, \quad g_{3}=0, \quad g_{4}=-g_{2}, \quad g_{5}=-g_{1}, \quad g_{6}=-g_{0}, \\
f_{0}=-\frac{\sqrt{2}}{6}, \quad f_{1}=\frac{\sqrt{2}}{6}, \quad f_{2}=f_{1}, \quad f_{3}=f_{0}, \\
s_{0}=\frac{\sqrt{2}}{18}, \quad s_{1}=-\frac{5 \sqrt{2}}{18}, \quad s_{2}=-s_{1}, \quad s_{3}=-s_{0},
\end{array}
$$

The resulting symmetric refinable function $\phi \in W^{0.8155}(R)$. See Figure 1 for the graphs of $\phi$, $\psi^{1}, \psi^{2}$, and $\psi^{3}$. 


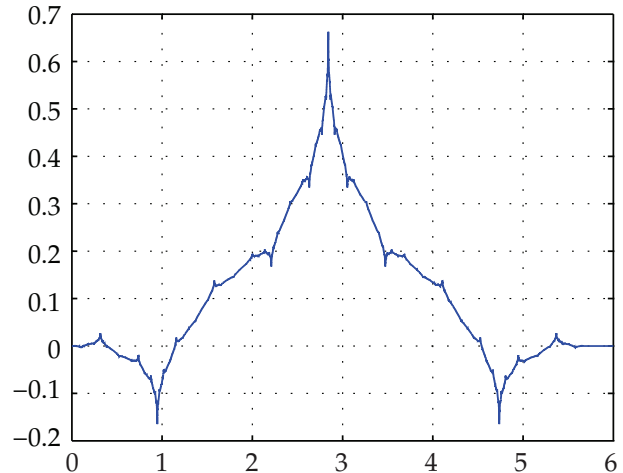

(a)

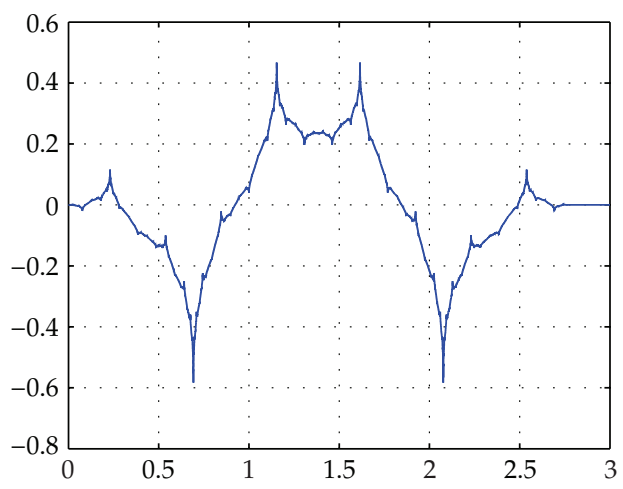

(c)

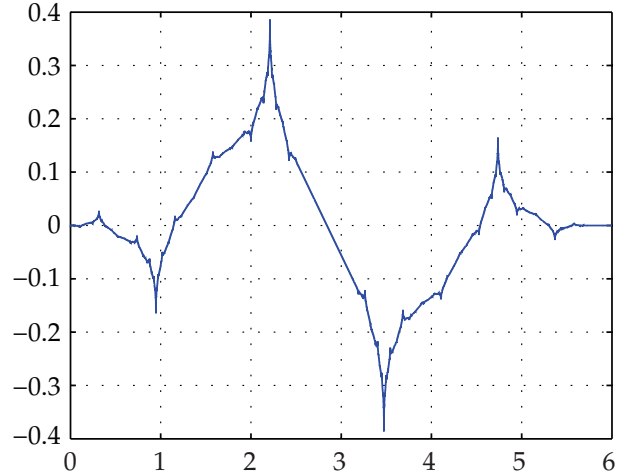

(b)

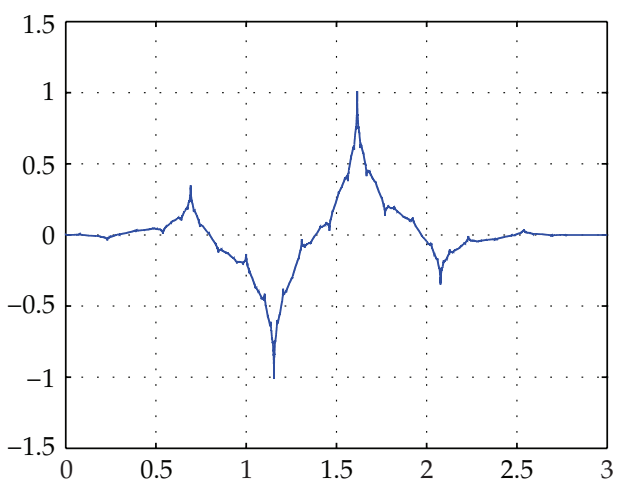

(d)

Figure 1: (a) Refinable function $\phi \in W^{0.8155}(R)$ with symmetry, (b) framelets $\psi^{1}$ with antisymmetry, and (c) and (d) $\psi^{2}$ symmetry and $\psi^{3}$ with antisymmetry, respectively.

Example 5.2. Let $h, g, f, s$ be the filters given by (4.26) with $n=1$. For $h(1)=1, g(1)=f(1)=$ $s(1)=0, h(k)=0, k=-1 / 2 \pm(\sqrt{3} / 2) i$, let $a_{1}=\sin \theta, a_{2}=\cos \theta, b_{1}=\sin \theta_{1}, b_{2}=\cos \theta_{1}$, $c_{1}=\sin \theta_{2}, c_{2}=\cos \theta_{2}$, then we have

$$
\begin{aligned}
& a_{1}=-\frac{1}{6}\left[-2 \sqrt{2} b_{2}^{2}+8 \sqrt{2} b_{2} \sqrt{1-b_{2}^{2}}-4 b_{2}^{2}+8 b_{2} \sqrt{1-b_{2}^{2}}+2+\frac{\sqrt{6}}{3 b_{2}}+\sqrt{1-b_{2}^{2}}+2 \sqrt{2} b_{2}\right], \\
& a_{2}=-\frac{\sqrt{6}}{6}\left(-b_{2}+\sqrt{1-b_{2}^{2}}-\sqrt{2} b_{2}-\sqrt{2-2 b_{2}^{2}}\right) \\
& b_{1}=\sqrt{1-b_{2}^{2}}, \quad b_{2}=b_{2}, \\
& c_{1}= \pm \sqrt{1-c_{2}^{2}}, \quad c_{2}=c_{2} .
\end{aligned}
$$

Let $h$ has sum rules of order 2, such as $h^{\prime}(k)=0$, then we can get that $\sin \theta=-0.8625$, $\cos \theta=-0.5061, \sin \alpha_{1}=0.7645, \cos \alpha_{1}=-0.6447, \sin \alpha_{2}=-0.5172, \cos \alpha_{2}=-0.8559$. 


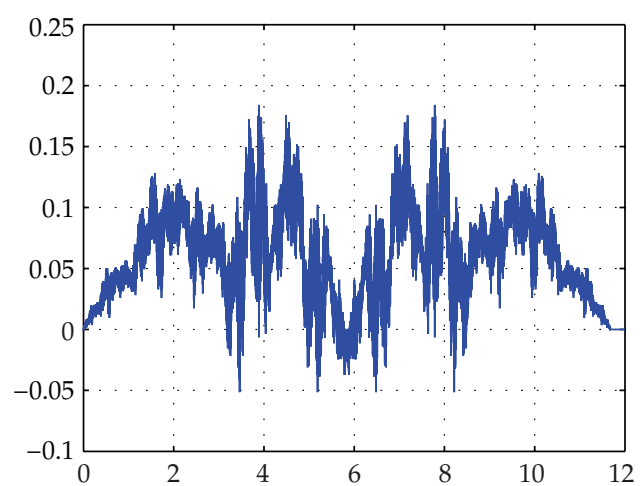

(a)

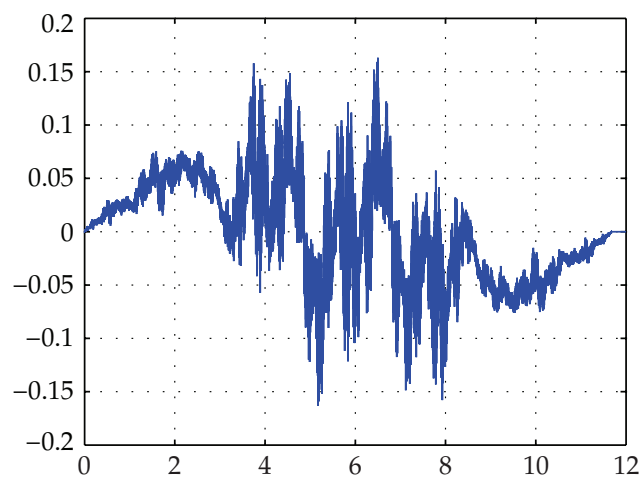

(c)

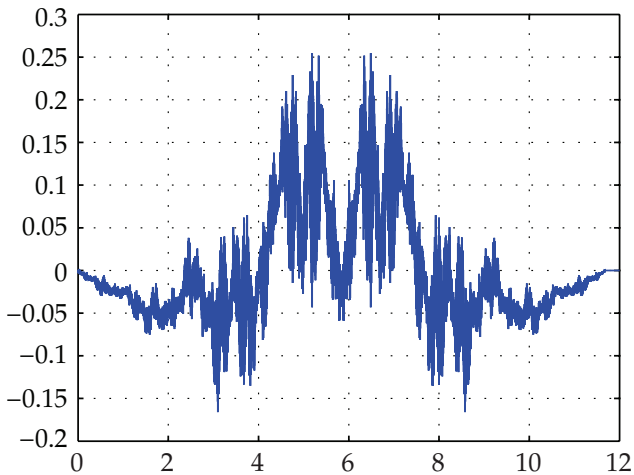

(b)

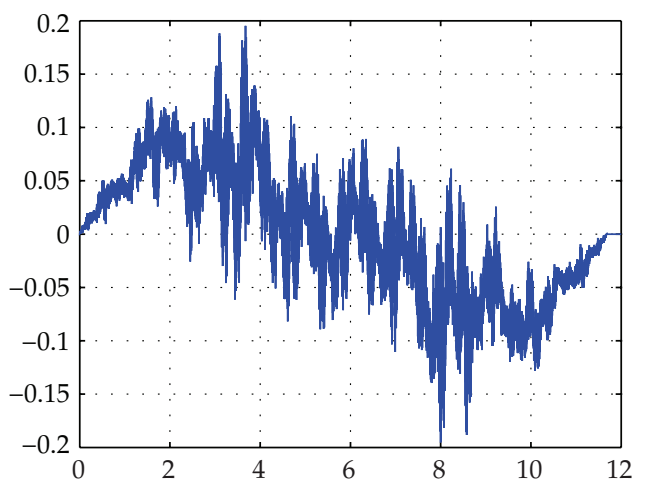

(d)

Figure 2: (a) Refinable function $\phi \in W^{0.3048}(R)$ with symmetry, (b) framelets $\psi^{1}$ with symmetry, and (c) and (d) $\psi^{2}$ symmetry and $\psi^{3}$ with antisymmetry, respectively.

Thus

$$
\begin{array}{ll}
h_{0}=0.1314, & h_{1}=0.1333, \quad h_{2}=0.2176, \quad h_{3}=-0.0550, \quad h_{4}=-0.1091, \\
h_{5}=0.0915, & h_{6}=0.1806, \quad h_{7}=h_{5}, \ldots, h_{12}=h_{0}, \\
g_{0}=-0.0771, \quad g_{1}=-0.0782, \quad g_{2}=-0.1277, \quad g_{3}=0.1590, \quad g_{4}=-0.1858, \\
g_{5}=0.1560, \quad h_{6}=0.3077, \quad g_{7}=g_{5}, \ldots, g_{12}=g_{0}, \\
f_{0}=0.0771, \quad f_{1}=0.0782, \quad f_{2}=0.1277, \quad f_{3}=0.0295, \quad f_{4}=-0.1058, \\
f_{5}=0.1560, \quad f_{6}=0, \quad f_{7}=-f_{5}, \ldots, f_{12}=-f_{0}, \\
s_{0}=0.1314, \quad s_{1}=0.1333, \quad s_{2}=0.2176, \quad s_{3}=-0.1656, \quad s_{4}=0.1091, \\
s_{5}=-0.0915, \quad s_{6}=0, \quad s_{7}=-s_{5}, \ldots, s_{12}=-s_{0} .
\end{array}
$$

The resulting symmetric refinable function $\phi \in W^{0.3048}(R)$. See Figure 2 for the graphs of $\phi$, $\psi^{1}, \psi^{2}$, and $\psi^{3}$. 


\section{Acknowledgments}

The authors would like to thank the support of the National Natural Science Foundation of China (Grant no. 61272028). The authors also appreciate the support of the Fundamental Research Funds for the Central Universities (no. ZZ1229), National Undergraduate Training Programs for Innovation and Entrepreneurship (no. 201210010077) and Science and Technology Innovation Foundation for the College Students (no. pt2012064).

\section{References}

[1] G. Strang and T. Nguyen, Wavelets and Filter Banks, Wellesley-Cambridge Press, Wellesley, Mass, USA, 1996.

[2] T. Xia and Q. Jiang, "Optimal multifilter banks: design, related symmetric extension transform, and application to image compression," IEEE Transactions on Signal Processing, vol. 47, no. 7, pp. 1878-1889, 1999.

[3] A. K. Soman, P. P. Vaidyanathan, and T. Q. Nguyen, "Linear phase paraunitary filter banks: theory, factorizations and designs," IEEE Transactions on Signal Processing, vol. 41, no. 12, pp. 3480-3496, 1993.

[4] C. K. Chui and W. He, "Compactly supported tight frames associated with refinable functions," Applied and Computational Harmonic Analysis, vol. 8, no. 3, pp. 293-319, 2000.

[5] I. W. Selesnick, "Smooth wavelet tight frames with zero moments," Applied and Computational Harmonic Analysis, vol. 10, no. 2, pp. 163-181, 2001.

[6] C. K. Chui, W. He, and J. Stöckler, "Compactly supported tight and sibling frames with maximum vanishing moments," Applied and Computational Harmonic Analysis, vol. 13, no. 3, pp. 224-262, 2002.

[7] I. Daubechies, B. Han, A. Ron, and Z. Shen, "Framelets: MRA-based constructions of wavelet frames," Applied and Computational Harmonic Analysis, vol. 14, no. 1, pp. 1-46, 2003.

[8] C. K. Chui, W. He, J. Stöckler, and Q. Sun, "Compactly supported tight affine frames with integer dilations and maximum vanishing moments," Advances in Computational Mathematics, vol. 18, no. 2-4, pp. 159-187, 2003.

[9] Q. Jiang, "Parameterizations of masks for tight affine frames with two symmetric/antisymmetric generators," Advances in Computational Mathematics, vol. 18, no. 2-4, pp. 247-268, 2003.

[10] A. Petukhov, "Symmetric framelets," Constructive Approximation, vol. 19, no. 1, pp. 309-328, 2003.

[11] I. W. Selesnick and A. F. Abdelnour, "Symmetric wavelet tight frames with two generators," Applied and Computational Harmonic Analysis, vol. 17, no. 2, pp. 211-225, 2004.

[12] B. Han and Q. Mo, "Symmetric MRA tight wavelet frames with three generators and high vanishing moments," Applied and Computational Harmonic Analysis, vol. 18, no. 1, pp. 67-93, 2005.

[13] R. H. Chan, Z. Shen, and T. Xia, "A framelet algorithm for enhancing video stills," Applied and Computational Harmonic Analysis, vol. 23, no. 2, pp. 153-170, 2007.

[14] J.-F. Cai, R. H. Chan, and Z. Shen, "A framelet-based image inpainting algorithm," Applied and Computational Harmonic Analysis, vol. 24, no. 2, pp. 131-149, 2008.

[15] B. Dong, A. Chien, and Z. Shen, "Frame based segmentation for medical images," Communications in Mathematical Sciences, vol. 9, no. 2, pp. 551-559, 2011.

[16] A. Ron and Z. Shen, "Affine systems in $L_{2}\left(R^{d}\right)$ : the analysis of the analysis operator," Journal of Functional Analysis, vol. 148, no. 2, pp. 408-447, 1997.

[17] A. Ron and Z. Shen, "Affine systems in $L_{2}\left(R^{d}\right)$. II. Dual systems," The Journal of Fourier Analysis and Applications, vol. 3, no. 5, pp. 617-637, 1997.

[18] P. Steffen, P. N. Heller, R. A. Gopinath, and C. S. Burrus, "Theory of regular M-band wavelet bases," IEEE Transactions on Signal Processing, vol. 41, no. 12, pp. 3497-3511, 1993.

[19] Q. Jiang, "Parameterization of $m$-channel orthogonal multifilter banks," Advances in Computational Mathematics, vol. 12, no. 2-3, pp. 189-211, 2000.

[20] L. Cui and R. Cong, "Construction for a class of interpolation multiscaling functions with dilation factor $a \geq 3$," Computers $\mathcal{E}$ Mathematics with Applications, vol. 56, no. 11, pp. 2948-2956, 2008.

[21] L. Cui and T. Zhang, " $m$-band orthogonal vector-valued multiwavelets for vector-valued signals," Journal of Applied Mathematics and Computing, vol. 28, no. 1-2, pp. 165-184, 2008.

[22] L. H. Cui, Y. Huang, and J. J. Sun, "Parameterizations of masks for 3-band tight wavelet frames by symmetric extension of polyphase matrix," submitted to Applied Mathematics and Computation. 


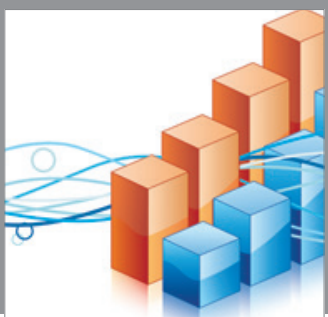

Advances in

Operations Research

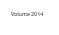

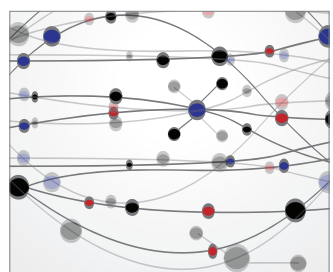

\section{The Scientific} World Journal
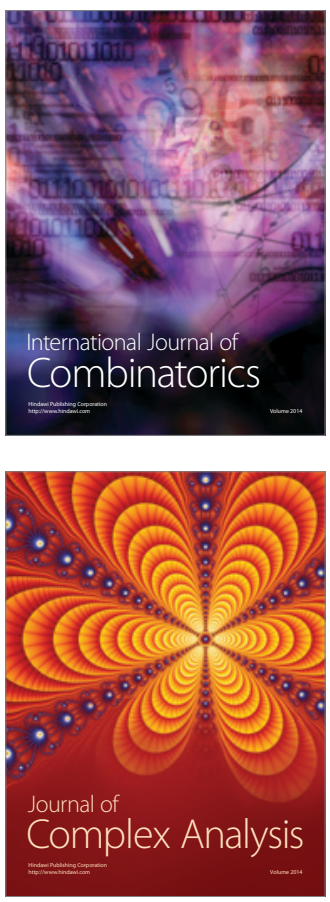

International Journal of

Mathematics and

Mathematical

Sciences
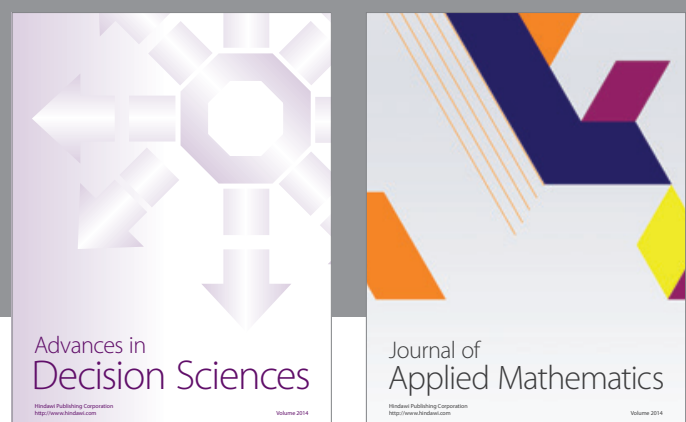

Journal of

Applied Mathematics
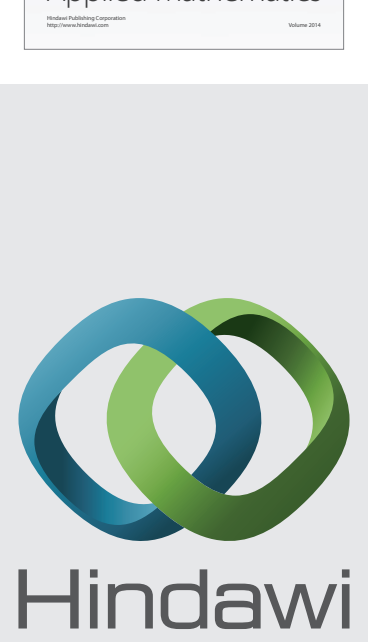

Submit your manuscripts at http://www.hindawi.com
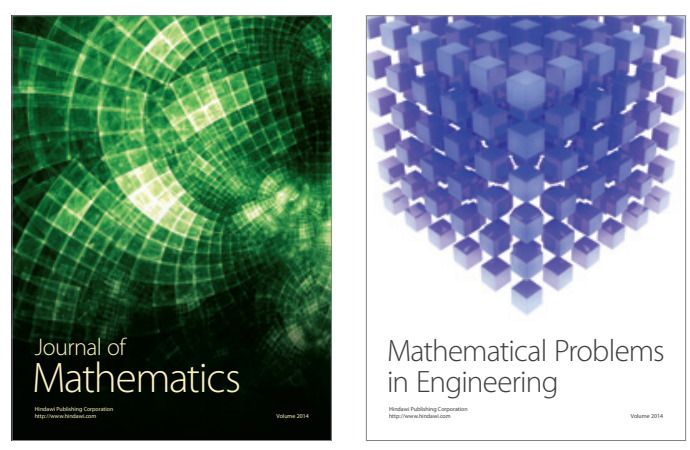

Mathematical Problems in Engineering
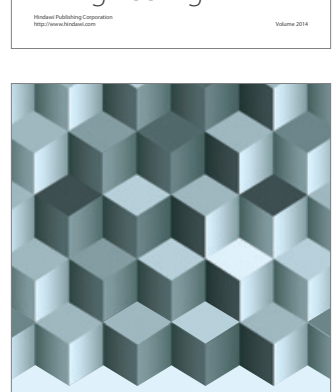

Journal of

Function Spaces
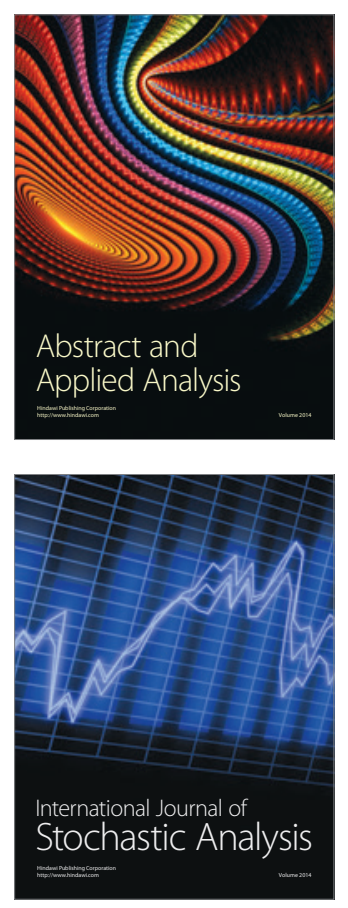

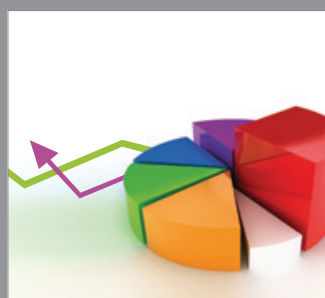

ournal of

Probability and Statistics

Promensencen
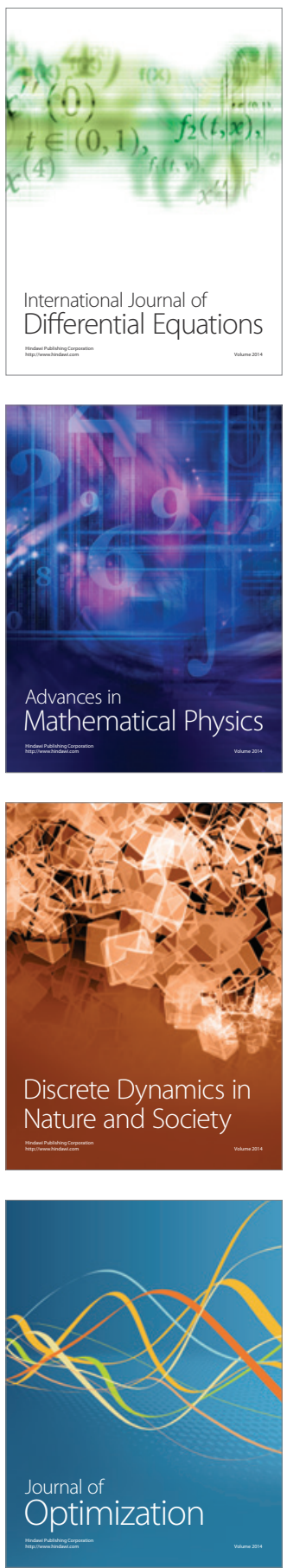